RAMOS, O.S. et al. Levantamento quantitativo de artigos sobre endoparasitismo em equinos publicados em periódicos na área de ciências agrárias nos últimos 10 anos no Brasil. PUBVET, Londrina, V. 8, N. 6, Ed. 255, Art. 1690, Março, 2014.

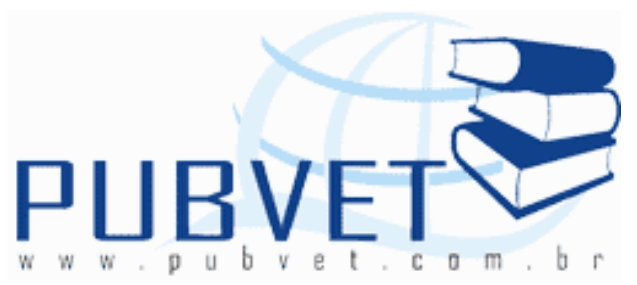

PUBVET, Publicações em Medicina Veterinária e Zootecnia.

\title{
Levantamento quantitativo de artigos sobre endoparasitismo em equinos publicados em periódicos na área de ciências agrárias nos últimos 10 anos no Brasil
}

Orllyane Santos Ramos ${ }^{1}$, Osvaldo José da Silveira Neto², Rodrigo Zaiden Taveira $^{2}$, Bruna Cristhina de Oliveira ${ }^{3}$, Taynara Raimundo Martins ${ }^{3}$

${ }^{1}$ Acadêmica do curso de Zootecnia da Pontifícia Universidade Católica de Goiás, Goiânia-GO

${ }^{2}$ Docente e Pesquisador - Curso de Zootecnia - UEG - UnU - São Luís de Montes Belos.

${ }^{3}$ Acadêmica do curso de Zootecnia da Universidade Estadual de Goiás, São Luís de Montes Belos - Brasil

\section{Resumo}

Foi realizado levantamento bibliográfico quantitativo acerca das publicações de artigos científicos referentes ao endoparasitismo e suas implicações nos equinos, nos principais periódicos nacionais na área de Ciências Agrárias. Foram selecionados os periódicos de circulação nacional, que obtiveram os conceitos A e B nas áreas de Ciências Agrárias I, Zootecnia, e Medicina Veterinária na avaliação do Qualis. Foram considerados os trabalhos publicados no período de 2002 a 2011, obtidos por meio de busca eletrônica. Foram encontrados 26 artigos que atenderam os requisitos especificados nesse estudo, sendo que a Revista Brasileira de Parasitologia Veterinária foi a que 
RAMOS, O.S. et al. Levantamento quantitativo de artigos sobre endoparasitismo em equinos publicados em periódicos na área de ciências agrárias nos últimos 10 anos no Brasil. PUBVET, Londrina, V. 8, N. 6, Ed. 255, Art. 1690, Março, 2014.

apresentou o maior número de publicações referentes ao tema. Considerando que as verminoses afetam diretamente o desempenho natural dos equinos, é importante realizar periodicamente o controle dos parasitas. Entretanto, o pouco interesse por parte da comunidade científica acerca do assunto dificulta a tomada de decisões dos criadores a respeito dos melhores métodos de controle helmíntico.

Palavras-chave: Cavalos, Controle helmíntico, Verminose.

\title{
Quantitative bibliographic study about the scientifically articles concerning to the parasitism in equines, in the mainly national scientific publications in the animal science
}

\begin{abstract}
It was carried out quantitative bibliographic study about the scientifically articles concerning to the parasitism and theirs implications in equines, in the mainly national scientific publications in the Animal Science. It was selected the publications of national circulation, that obtained grades $A$ and $B$ in the Animal Science I, Animal production, and veterinary in the Qualis evaluation. It was considered papers published form 2002 to 2011, obtained by electronic method. It was finding 26 articles according the requisites required, being the "Revista Brasileira de Parasitologia Veterinária" that showed the higher number of publication in this field. Considering that the verminous affect directly the equines natural performances is very important do the periodic parasites control. However, the less interest by the scientifically community in this area, become difficult the breeders decisions concerning the best methods for the verminous control.
\end{abstract}

Keywords: Horses, Helminthic control, Verminous 
RAMOS, O.S. et al. Levantamento quantitativo de artigos sobre endoparasitismo em equinos publicados em periódicos na área de ciências agrárias nos últimos 10 anos no Brasil. PUBVET, Londrina, V. 8, N. 6, Ed. 255, Art. 1690, Março, 2014.

\section{Introdução}

O rebanho equino brasileiro situa-se como o terceiro maior do mundo e o maior da América Latina, com cerca de 5.514.253 cabeças. O maior efetivo equino brasileiro encontra-se na região Nordeste, seguida pelas regiões Sudeste, Centro-Oeste, Sul e Norte. Dentre os estados, Minas Gerais é o que possui a maior população equina do país, com 802.049 cabeças, sendo Corumbá - MS o município como maior efetivo equino do Brasil, com 29.890 cabeças (IBGE, 2010).

O complexo do agronegócio equino no Brasil movimenta cerca de $R \$ 7,5$ bilhões e gera cerca de 3,2 milhões de empregos diretos e indiretos. 0 equino, no aspecto econômico, desempenha funções de sela, carga e tração e a partir da segunda metade do século $X X$, tem se destacado no aspecto social as atividades de esportes e lazer (LIMA et al., 2006).

Tendo em vista os sistemas de criação e os hábitos alimentares, os equinos são altamente susceptíveis às infecções parasitárias. Eles se contaminam por meio do próprio ambiente em que vivem, sejam pastagens ou baias. As verminoses são apontadas como uma das principais causas do baixo rendimento zootécnico dos rebanhos e mortes precoces em animais, ainda em fase produtiva, dessa forma é importante realizar o seu controle a fim de se obter melhor desempenho dos animais.

Os equinos apresentam grande variedade de parasitas em sua fauna helmíntica, e algumas espécies/gêneros são de grande importância, como: Parascaris equorum, Anoplocephala perfoliata, Oxyuris equi, Cyathostomum spp. e Strongylus spp. (MOLENTO, 2005).

Os helmintos que acometem os equinos afetam diretamente seu desenvolvimento, causando diversas doenças, como aneurisma verminótico, gastroenterite e dermatites. Os animais parasitados podem apresentar fraqueza, retardo no crescimento e até episódios fulminantes de cólicas e morte. 
RAMOS, O.S. et al. Levantamento quantitativo de artigos sobre endoparasitismo em equinos publicados em periódicos na área de ciências agrárias nos últimos 10 anos no Brasil. PUBVET, Londrina, V. 8, N. 6, Ed. 255, Art. 1690, Março, 2014.

Nos plantéis que realizam desverminação, o controle do parasitismo é realizado por meio de compostos anti-helmínticos, na forma de pasta, em virtude da praticidade, eficiência e segurança. Porém, o uso indiscriminado desses compostos e a ausência de medidas de higiene podem resultar no desenvolvimento de resistência por parte dos parasitas.

Existem poucos trabalhos disponíveis na literatura brasileira sobre o nível de infestação e avaliação do melhor princípio ativo no combate ao parasitismo em equinos, o que gera escassez no acervo bibliográfico dessa temática, dificultando a tomada de decisões por parte dos criadores quanto ao controle de endoparasitas respaldado por embasamento científico.

Sendo assim, objetivou-se realizar levantamento bibliográfico quantitativo acerca das publicações de artigos científicos referentes ao endoparasitismo e suas implicações nos equinos, nos principais periódicos nacionais na área de Ciências Agrárias, a fim de comprovar a escassez de trabalhos dessa natureza.

\section{Material e métodos}

O material para desenvolvimento desse estudo constitui-se de artigos publicados nos principais periódicos nacionais na área de Ciências Agrárias, os quais são discriminados na TABELA 1.

Utilizou-se como critério de escolha dos periódicos a serem pesquisados a avaliação, no ano de 2012, pelo sistema de classificação de periódicos Qualis, divulgado pela Coordenação de Aperfeiçoamento de Pessoal de Nível Superior (CAPES).

Foram selecionados os periódicos de circulação nacional que obtiveram conceitos A e B nas áreas de Ciências Agrárias I, Zootecnia e Medicina Veterinária, tal classificação segue na TABELA 2. 
RAMOS, O.S. et al. Levantamento quantitativo de artigos sobre endoparasitismo em equinos publicados em periódicos na área de ciências agrárias nos últimos 10 anos no Brasil. PUBVET, Londrina, V. 8, N. 6, Ed. 255, Art. 1690, Março, 2014.

TABELA 1: Periódicos na área de Ciências Agrárias selecionados para o estudo.

\begin{tabular}{|c|c|c|c|}
\hline Periódicos & Editor & $\begin{array}{l}\text { Periodicidad } \\
\text { e Atual }\end{array}$ & Início \\
\hline $\begin{array}{l}\text { Revista Brasileira } \\
\text { de Zootecnia }\end{array}$ & $\begin{array}{l}\text { Sociedade Brasileira de } \\
\text { Zootecnia }\end{array}$ & Mensal & 1972 \\
\hline $\begin{array}{l}\text { Revista Brasileira } \\
\text { de Parasitologia } \\
\text { Veterinária }\end{array}$ & $\begin{array}{l}\text { Colégio Brasileiro de } \\
\text { Parasitologia Veterinária }\end{array}$ & Trimestral & 1992 \\
\hline Ciência Rural & $\begin{array}{l}\text { Universidade Federal de } \\
\text { Santa Maria }\end{array}$ & Bimestral & 1971 \\
\hline $\begin{array}{l}\text { Ciência e } \\
\text { Agrotecnologia }\end{array}$ & $\begin{array}{l}\text { Universidade Federal de } \\
\text { Lavras }\end{array}$ & Bimestral & 1977 \\
\hline $\begin{array}{l}\text { Arquivo Brasileiro } \\
\text { de Medicina } \\
\text { Veterinária e } \\
\text { Zootecnia }\end{array}$ & $\begin{array}{l}\text { Universidade Federal de } \\
\text { Minas Gerais, Escola de } \\
\text { Veterinária }\end{array}$ & Bimestral & 1943 \\
\hline $\begin{array}{l}\text { Pesquisa } \\
\text { Agropecuária } \\
\text { Brasileira }\end{array}$ & $\begin{array}{l}\text { Empresa Brasileira de } \\
\text { Pesquisa Agropecuária- } \\
\text { Embrapa }\end{array}$ & Mensal & 1966 \\
\hline $\begin{array}{l}\text { Pesquisa } \\
\text { Veterinária } \\
\text { Brasileira }\end{array}$ & $\begin{array}{l}\text { Colégio Brasileiro de } \\
\text { Patologia Animal }\end{array}$ & Mensal & 1981 \\
\hline $\begin{array}{l}\text { Semina: Ciências } \\
\text { Agrárias }\end{array}$ & $\begin{array}{l}\text { Universidade Estadual de } \\
\text { Londrina }\end{array}$ & Trimestral & 1978 \\
\hline Revista Caatinga & $\begin{array}{l}\text { Universidade Federal Rural } \\
\text { do Semiárido }\end{array}$ & Trimestral & 1976 \\
\hline Revista Ceres & $\begin{array}{l}\text { Universidade Federal de } \\
\text { Viçosa }\end{array}$ & Bimestral & 1939 \\
\hline $\begin{array}{l}\text { Ciência Animal } \\
\text { Brasileira }\end{array}$ & $\begin{array}{l}\text { Universidade Federal de } \\
\text { Goiás }\end{array}$ & Trimestral & 2000 \\
\hline ARS Veterinária & $\begin{array}{l}\text { Universidade Estadual } \\
\text { Paulista }\end{array}$ & Trimestral & 1985 \\
\hline
\end{tabular}


RAMOS, O.S. et al. Levantamento quantitativo de artigos sobre endoparasitismo em equinos publicados em periódicos na área de ciências agrárias nos últimos 10 anos no Brasil. PUBVET, Londrina, V. 8, N. 6, Ed. 255, Art. 1690, Março, 2014.

TABELA 2: Classificação dos periódicos, segundo o Qualis, nas áreas de Ciências Agrárias I, Zootecnia e Medicina Veterinária.

\begin{tabular}{lccc}
\hline \multicolumn{1}{c}{ Periódicos } & $\begin{array}{c}\text { Classificação } \\
\text { Ciências } \\
\text { Agrárias I }\end{array}$ & Zootecnia & $\begin{array}{c}\text { Medicina } \\
\text { Veterinária }\end{array}$ \\
\hline Revista Brasileira de Zootecnia & B1 & B1 & B1 \\
$\begin{array}{l}\text { Revista Brasileira de } \\
\text { Parasitologia Veterinária }\end{array}$ & B3 & B1 & B1 \\
Ciência Rural & B1 & B2 & B1 \\
Ciência e Agrotecnologia & B1 & B1 & - \\
$\begin{array}{l}\text { Arquivo Brasileiro de Medicina } \\
\text { Veterinária e Zootecnia }\end{array}$ & B1 & B2 & A2 \\
$\begin{array}{l}\text { Pesquisa Agropecuária } \\
\text { Brasileira }\end{array}$ & B1 & B1 & B2 \\
Pesquisa Veterinária Brasileira & B2 & B1 & A2 \\
Semina: Ciências Agrárias & B1 & B2 & B1 \\
Revista Caatinga & B2 & B4 & B5 \\
Revista Ceres & B2 & B4 & B3 \\
Ciência Animal Brasileira & B5 & B4 & B2 \\
ARS Veterinária & B5 & B5 & B4 \\
\hline
\end{tabular}

Foram considerados os trabalhos publicados no período de 2002 a 2011 , referentes ao endoparasitismo em equinos, obtidos por meio de busca eletrônica, pela versão online dos periódicos. 
RAMOS, O.S. et al. Levantamento quantitativo de artigos sobre endoparasitismo em equinos publicados em periódicos na área de ciências agrárias nos últimos 10 anos no Brasil. PUBVET, Londrina, V. 8, N. 6, Ed. 255, Art. 1690, Março, 2014.

\section{Resultados e discussão}

A TABELA 3 dada abaixo apresenta a distribuição dos artigos publicados por periódico ao longo dos dez anos da avaliação realizada.

TABELA 3 - Publicações de artigos sobre endoparasitismo em equinos nos periódicos nacionais na área de Ciências Agrárias no ano de 2002 a 2011.

\begin{tabular}{|c|c|c|c|c|c|c|c|c|c|c|c|c|}
\hline Periódicos & & 2002 & 03 & 04 & 05 & 06 & 07 & 08 & 09 & 10 & 2011 & Total \\
\hline $\begin{array}{l}\text { Revista Brasileira de } \\
\text { Zootecnia }\end{array}$ & & - & - & - & - & - & - & - & - & - & - & 0 \\
\hline $\begin{array}{l}\text { Revista Brasileira de } \\
\text { Parasitologia Veterinária }\end{array}$ & & 1 & 3 & - & - & - & - & 4 & 2 & - & 1 & 11 \\
\hline Ciência Rural & & 1 & - & - & 1 & - & 1 & 1 & - & - & - & 4 \\
\hline Ciência e Agrotecnologia & & - & - & - & - & - & - & - & - & - & - & 0 \\
\hline $\begin{array}{l}\text { Arquivo Brasileiro de } \\
\text { Medicina Veterinária e } \\
\text { Zootecnia }\end{array}$ & & - & - & - & 1 & - & - & - & - & - & - & 1 \\
\hline $\begin{array}{l}\text { Pesquisa Agropecuária } \\
\text { Brasileira }\end{array}$ & & - & - & - & - & - & - & - & - & - & - & 0 \\
\hline $\begin{array}{l}\text { Pesquisa Veterinária } \\
\text { Brasileira }\end{array}$ & & & & & & & & & & & & \\
\hline & & - & 1 & - & - & - & - & - & 2 & - & - & 3 \\
\hline Semina: Ciências Agrárias & & - & - & - & - & - & - & 1 & 1 & - & - & 2 \\
\hline Revista Caatinga & $*$ & $*$ & * & & * & - & - & 1 & - & - & - & 1 \\
\hline Revista Ceres & - & - & - & & - & - & - & - & - & - & - & 0 \\
\hline Ciência Animal Brasileira & - & - & - & & - & - & 1 & - & 1 & 2 & - & 4 \\
\hline ARS Veterinária & - & - & - & & - & - & - & - & - & - & - & 0 \\
\hline Total & 2 & 4 & 0 & & 2 & כ & 2 & 7 & 6 & 2 & 1 & 26 \\
\hline
\end{tabular}

*Não estão disponíveis na versão online as publicações de 2002 a 2005. 
RAMOS, O.S. et al. Levantamento quantitativo de artigos sobre endoparasitismo em equinos publicados em periódicos na área de ciências agrárias nos últimos 10 anos no Brasil. PUBVET, Londrina, V. 8, N. 6, Ed. 255, Art. 1690, Março, 2014.

Foram encontrados 26 artigos que atenderam os requisitos especificados nesse estudo. Considerando todos os periódicos avaliados, constatou-se que cinco $(41,7 \%)$, sendo eles: Revista Brasileira de Zootecnia, Ciência e Agrotecnologia, Pesquisa Agropecuária Brasileira, Revista Ceres e ARS Veterinária, não publicaram nenhum artigo a respeito do tema pesquisado. Esse registro demonstra que houve pouca procura para publicação de artigos relacionados à verminose equina em periódicos menos especializados nessa área.

A Revista Brasileira de Parasitologia Veterinária foi a que apresentou maior número de publicações referentes ao tema, tendo em vista a especialidade de seu escopo. Entretanto, percebe-se que a espécie equina ainda é objeto de pouco estudo dentro dessa temática, quando comparada às outras espécies.

Por meio da análise dos artigos publicados, percebeu-se que todos poderiam ser enquadrados basicamente em três categorias, sendo: avaliação da eficácia de anti-helmínticos; métodos alternativos de controle de verminoses e caracterização, infestação e prevalência de espécies de helmintos, o que pode ser observado na TABELA 4.

Apenas quatro artigos avaliaram a eficácia de anti-helmínticos no controle de endoparasitoses, sendo que em todos foi testada a eficácia das lactonas macrocíclicas, um dos antiparasitários mais utilizados atualmente. Esses registros denotam a pouca importância destinada aos estudos envolvendo o controle helmíntico nos equinos. O grupo das lactonas foi envolvido em todos os estudos, provavelmente, por apresentar amplo espectro de ação e por terem poucos relatos de resistência dos parasitas a esse princípio ativo.

Dos artigos sobre métodos alternativos de controle, sete referem-se ao controle biológico, através do uso de fungos nematófagos, demonstrando que há interesse por parte dos pesquisadores em encontrar outros métodos associados de controle de helmintos em equinos. 
RAMOS, O.S. et al. Levantamento quantitativo de artigos sobre endoparasitismo em equinos publicados em periódicos na área de ciências agrárias nos últimos 10 anos no Brasil. PUBVET, Londrina, V. 8, N. 6, Ed. 255, Art. 1690, Março, 2014.

TABELA 4 - Quantificação de publicações de sete periódicos relacionados à verminose na espécie equina em função da área específica de estudo.

\begin{tabular}{|c|c|c|c|c|}
\hline \multirow{2}{*}{\multicolumn{2}{|c|}{ Periódicos }} & \multicolumn{3}{|c|}{ Categorias } \\
\hline & & \multirow{2}{*}{$\begin{array}{c}\text { A* } \\
1\end{array}$} & \multirow{2}{*}{$\begin{array}{c}\text { B* } \\
3\end{array}$} & \multirow{2}{*}{$\begin{array}{c}\text { C* } \\
7 \\
7\end{array}$} \\
\hline $\begin{array}{l}\text { Revista Brasileira de } \\
\text { Veterinária }\end{array}$ & Parasitologia & & & \\
\hline \multicolumn{2}{|l|}{ Ciência Rural } & 1 & 1 & 2 \\
\hline \multicolumn{2}{|c|}{$\begin{array}{l}\text { Arquivo Brasileiro de Medicina Veterinária e } \\
\text { Zootecnia }\end{array}$} & - & - & 1 \\
\hline \multicolumn{2}{|l|}{ Pesquisa Veterinária Brasileira } & - & 2 & 1 \\
\hline \multicolumn{2}{|l|}{ Semina: Ciências Agrárias } & - & - & 2 \\
\hline \multicolumn{2}{|l|}{ Revista Caatinga } & 1 & - & - \\
\hline \multicolumn{2}{|l|}{ Ciência Animal Brasileira } & 1 & 1 & 2 \\
\hline \multicolumn{2}{|l|}{ Total } & 4 & 7 & 15 \\
\hline \multicolumn{5}{|c|}{ A*- Avaliação da eficácia de anti-helmínticos } \\
\hline \multicolumn{5}{|c|}{ B*- Métodos alternativos de controle de verminoses } \\
\hline \multicolumn{5}{|c|}{ C*- Caracterização, infestação e prevalência de espécies de helmintos } \\
\hline
\end{tabular}

A infecção por ciatostomíneos, os pequenos estrongilos, foi registrada em quinze artigos publicados, o que demonstra a susceptibilidade dos equinos a essa classe de vermes, os quais conforme Molento (2005) são os parasitas mais prevalentes e resistentes a anti-helmínticos.

Tendo em vista a escassez de recursos destinados às pesquisas, o equino, muitas vezes, é relegado a segundo plano, em detrimento das espécies diretamente ligadas à produção de proteína. Entretanto, deve ser considerado que o mesmo representa importante elemento dentro dos sistemas produtores, devendo, portanto, ser considerado em estudos que visem à maximização de seu desempenho. 
RAMOS, O.S. et al. Levantamento quantitativo de artigos sobre endoparasitismo em equinos publicados em periódicos na área de ciências agrárias nos últimos 10 anos no Brasil. PUBVET, Londrina, V. 8, N. 6, Ed. 255, Art. 1690, Março, 2014.

\title{
4 Conclusão
}

O levantamento quantitativo de artigos publicados nos últimos dez anos sobre endoparasitismo e suas implicações nos equinos, comprovou a escassez de trabalhos dessa natureza disponíveis na literatura brasileira.

Considerando que as verminoses afetam diretamente o desempenho natural dos equinos, é importante realizar periodicamente o controle dos parasitas. Entretanto, o pouco interesse por parte da comunidade científica acerca do assunto dificulta a tomada de decisões dos criadores a respeito dos melhores métodos de controle helmíntico.

\section{Referências bibliográficas}

\author{
InStituto bRASileiro de geografia e eStATÍsticA. Produção da Pecuária Municipal. \\ Rio de Janeiro: IBGE, 2010.2 Disponível \\ <http://www.ibge.gov.br/home/estatistica/economia/ppm/2010/ppm2010.pdf>. Acesso em: \\ 13 mar. 2012.
}

LIMA, R. A. S. et al. Estudo do complexo do agronegócio cavalo. Piracicaba: ESALQ/USP,2006. 250 p. Disponível em: <http://www.cepea.esalq.usp.br/ pdf/cavalo_completo.pdf>. Acesso: 13 mar. 2012.

MOLENTO, M. B. Resistência parasitária em helmintos de equídeos e propostas de manejo. Ciência Rural, Santa Maria, v. 35, n. 6, p. 1469-1477, nov./dez., 2005. Disponível em: <http://www.scielo.br/pdf/cr/v35n6/a41v35n6.pdf>. Acesso em: 13 mar. 2012. 